\title{
The relation between recycled radio pulsars and wide low-mass X-ray binaries
}

\author{
Xiangdong Li, Zhenru Wang \\ Department of Astronomy, Nanjing University, Nanjing, China
}

\section{Introduction}

The origin and evolution of neutron star magnetic fields has been hotly debated for a long time. Spontaneous field decay was originally proposed with timescales of $(5-10) \times 10^{6}$ years, while another possible model which associates field decay with mass accretion in the evolution of binary systems has been suggested (see Bhattacharya \& van den Heuvel 1991 for a review). The aim of this paper is to examine whether accretion-induced field decay can reproduce the observed properties of the wide binary radio pulsars in quantitative calculations.

\section{The model}

In a binary system consisting of a neutron star and a low-mass giant companion, if the initial orbital period is longer than 1 day, mass transfer, taking the form of Roche-lobe overflow, is driven by the nuclear evolution of the giant through radius expansion (Webbink et al. 1983). We assume the mass accretion rate $\dot{M}$ of the neutron star is limited to the Eddington accretion rate $\dot{M}_{\mathrm{E}} \simeq 10^{-8} M_{\odot}$ $\mathrm{yr}^{-1}$. If the mass transfer rate is in excess of $\dot{M}_{\mathrm{E}}$, the rest mass is blown from the system in the forms of jets or beams.

Instead of spontaneous field decay, we study the assumption of accretioninduced field decay to parametrize the magnetic field evolution. We adopt the following formula for field decay with mass accretion:

$$
B=B_{\mathrm{i}} /\left(1+\triangle M / m_{\mathrm{B}}\right)^{n},
$$

where $\triangle M$ is the accreted mass and $m_{\mathrm{B}}$ and $n$ are positive constants.

The spin period $P$ of the accreting neutron star will change due to the torque exerted on the neutron star by the accretion matter. An accretion disk is conventionally assumed to form around the neutron star. The spin evolution of the neutron star is calculated by use of the accretion torque models of Ghosh \& Lamb (1995), Yi (1995) and Li \& Wang (1995) respectively.

\section{Results}

We consider a binary consisting of a $1.3 M_{\odot}$ neutron star ánd a $0.8 M_{\odot}$ low-mass giant companion with heavy element abundance $Z=0.02$. We take the following initial values $B_{\mathrm{i}}=3 \times 10^{12} \mathrm{G}, P=5 \mathrm{sec}$, and $P_{\text {orb }}=1-500$ days. 

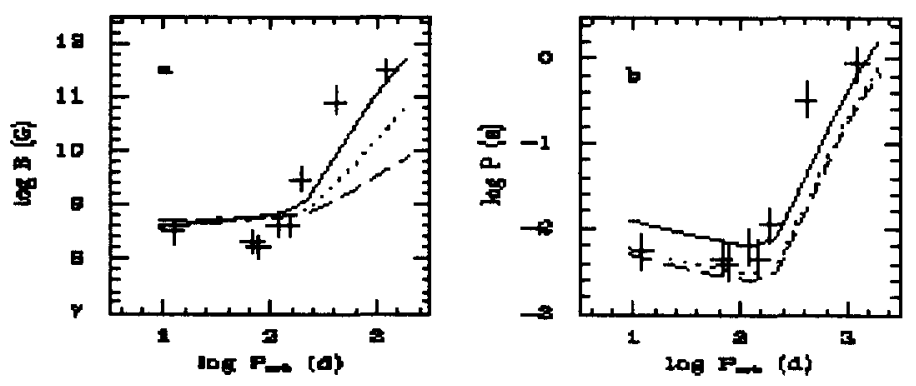

Figure 1. (a) The $B$ vs. $P_{\text {orb }}\left(\right.$ b) $P$ vs. $P_{\text {orb }}$ relations for wide, circular binary radio pulsars.

The calculated magnetic fields of the neutron star are plotted in Fig. 1a versus $P_{\text {orb. }}$. The solid, dotted and dashed lines represent the parameters $(n$, $\left.m_{\mathrm{B}} / M_{\odot}\right)$ being $(4,0.07),\left(2,7 \times 10^{-3}\right)$ and $\left(1,10^{-4}\right)$, respectively. The observed binary radio pulsars with wide, circular orbits (PSRs $0820+02,1620-26$, J16431224 , J1713+0747, 1800-27, 1855+09, 1953+29 and J2019+2425) are plotted with crosses. It is seen that only for $n \geq 3$ can the observations be fitted.

The spin period is also calculated and plotted in Fig. 1b, versus $P_{\text {orb }}$ with the observed binary radio pulsars shown for comparison. The parameters $n$ and $m_{\mathrm{B}}$ are taken to be 4 and $0.07 M_{\odot}$ respectively. The solid, dotted and dashed lines are the results calculated from the models of Ghosh \& Lamb (1979), Yi (1995) and Li \& Wang (1995) respectively.

\section{Conclusions}

The predicted results with a possible formula of accretion-induced field decay seem to be consistent with the observed $B$ vs. $P_{\text {orb }}$ and $P$ vs. $P_{\text {orb }}$ relations of wide binary radio pulsars. However, to account for the observations, a strong dependence of the magnetic field strength on mass accretion is required.

Acknowledgments. This work was supported by the National Natural Science Foundation of China.

\section{References}

Bhattacharya D., van den Heuvel E. P. J. 1991, Phys. Rep. 203, 1

Ghosh P., Lamb F. K. 1979, ApJ 234, 296

Li X.-D., Wang Z.-R. 1995, Ap\&SS 225, 47

Webbink R. F., Rappaport S. A., Savonije G. J. 1983, ApJ 270, 678

Yi I. 1995, ApJ 442, 786 